\title{
ADAPTIVE SUPPRESSION OF NARROW-BAND VIBRATIONS
}

\author{
Bertran, E. and G. Montoro \\ Department of Signal Theory and Communications \\ Universitat Politècnica de Catalunya \\ c/ Jordi Girona, 1-3 \\ 08034-Barcelona (Spain)
}

Phone: $34-93-4015938$

email: tscgml@eupbl.upc.es

\begin{abstract}
The aim of this paper is to develop a theoretical analysis and implementation of an active control canceller devoted to eliminate narrow-band vibrations in rotary machines. The proposed system uses a bank of digital adaptive notch filters each of them adjusted by an LMS algorithm. The theoretical results are applied to $a$ vibrating $D C$ motor considered as a benchmark problem. Experimental results reveal that a considerable vibration reduction is achievable.
\end{abstract}

\section{Introduction}

Vibrations occur frequently in rotary machines and usually are due to several mechanical problems such as: unbalanced mass, disadjustments in the components, wearing and ageing. Usually the machine vibration is characterized as a narrow-band signal, composed by subharmonic and harmonics of the machine speed (rotor frequency) [2],[5]. Sometimes a mechanical adjustment can reduce vibrations, but if the mechanical solution has a difficult implementation then control techniques (actives or passives) can be considered.

Passive and non-adaptive vibration cancellers have the disadvantage that complete narrow-band disturbance suppression needs an exact adjustment of their parameters. In fact, passive cancellers are inefficient for cancelling low frequencies as is the case of cancelling sub-harmonics [4].

Active control to cancel vibrations consist of generating a signal that must produce in the machine a vibration opposite to the undesired vibration, similar to a destructive interference: a counteracting signal is produced to bring the machine to a standstill. Therefore the waveform of the vibration must be known to succeed with active compensators. If vibrations are caused by rotary machinery with measurable speed it is feasible to keep track its frequency of rotation and this information can be used to identify the vibration wave.

The case of cancelling vibrations in a DC motor have a particular interest: it is a popular rotary system and it can be considered as a benchmark problem. The rotary machine (DC motor) is shown in the following figure:

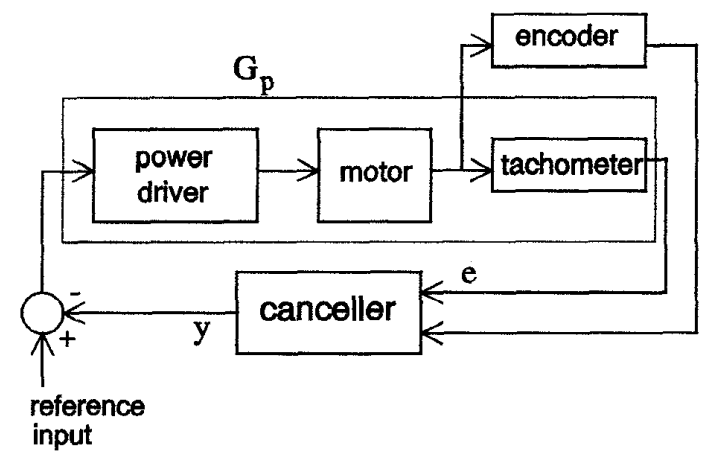

Fig. 1. Overall system: DC motor and canceller.

By sampling and processing the encoder signal it is possible to have a good measurement of the motor speed. This measurement is made by counting the zero-crossings of the square wave at the encoder output. Due to the zero mean of the random vibration, if the time-window is large enough then the zero crossing counting gives a good nominal motor speed. Moreover, a tachometer is employed to obtain the instantaneous motor speed (see Fig. 1). The noised tachometer measurement (due to vibrations) is used to search for a relationship between the nominal speed and the undesired vibration harmonics or sub-harmonics.

This relationship between nominal speed and the frequency of the vibration harmonics can be obtained 
from an spectral analysis of the tachometer signal. So, if this relation is known the next step is to excite the motor with an antivibration signal generated by the controller. This signal must be composed by a sum of sinusoids which frequencies correspond to ones of each harmonic and sub-harmonic to cancel, and which amplitude and phase must be correctly adjusted.

\section{Description of the Problem}

The problem is resumed in the following figure:

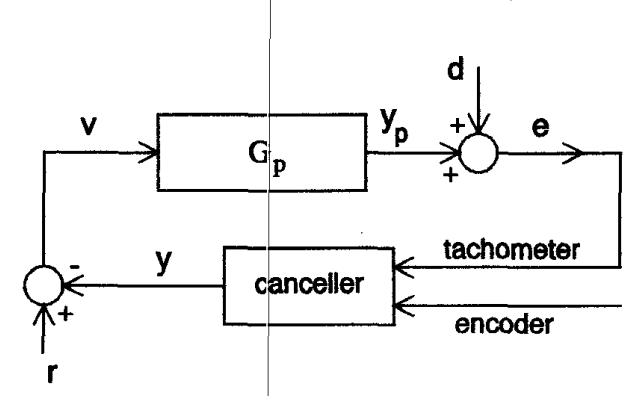

Fig. 2. Schematic representation.

The tachometer signal $e$ is composed by the response to the reference signal $r$, the vibration $d$ and the effect of the canceller. The measurement of the nominal motor speed is obtained processing the encoder signal (counting the zero crossings of the encoder signal).

The linear block $G_{p}$ represents the transfer function that characterizes the overall motor system composed by power driver, DC motor and tachometer.

For instance, if we consider the cancellation of subharmonics then the signal generated by the canceller $y$ will be composed by the sum of the sinusoids corresponding to the $N$ frequencies to cancel, such as:

$$
\begin{gathered}
y_{i}=A_{i} \cos \left(\omega_{i} t\right)+B_{i} \sin \left(\omega_{i} t\right)=\sqrt{A_{i}^{2}+B_{i}^{2}} \cos \left(\omega_{i} t-\varphi_{i}\right) \\
\omega_{i}=\frac{\omega_{p}}{N_{i}} \quad y=\sum_{i=1}^{N} y_{i}
\end{gathered}
$$

where each $N_{i}$ corresponds to one sub-harmonic of $\omega_{p}$ (the motor frequency). Considering the following formulation:

$$
e=d+G_{p}[r]-G_{p}[y]=x-G_{p}[y]
$$

where: $x=d+G_{p}[r]$

then the problem has an XLMS structure, such as:

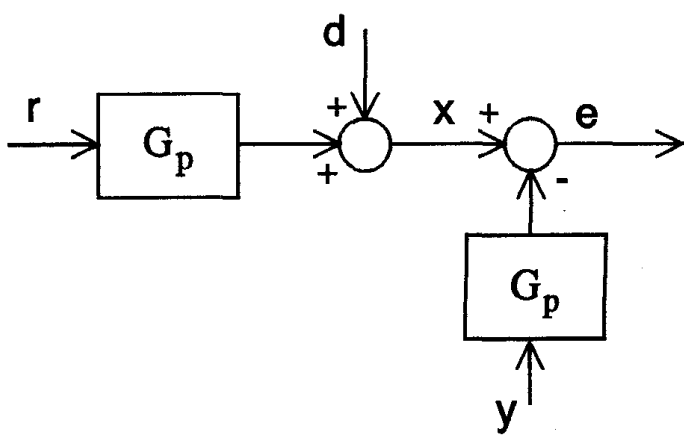

Fig. 3. XLMS structure.

\section{Adaptive Notch Canceller}

The antivibration method considered in this paper consist of exciting the motor with a signal composed by a sum of sinusoids (reference signals). Their frequencies correspond to each harmonic or sub-harmonic to be cancelled and their amplitudes and phases are adjusted by using and LMS algorithm. Therefore, the canceller works as a bank of adaptive notch filters, being each filter centered on an unwanted sinusoidal signal (vibration harmonics).

\subsection{Cancelling a Single Frequency}

The well-known formulation of a discrete-time adaptive notch canceller with sinusoidal reference is:

$$
y(n)=w_{1}(n) \cos \left(\omega_{0} n\right)+w_{2}(n) \sin \left(\omega_{0} n\right)
$$

where the two adaptive weights can be adjusted by using an LMS algorithm as:

$$
\begin{aligned}
& w_{1}(n+1)=w_{1}(n)+\mu_{c} e(n) \cos \left(\omega_{0} n\right) \\
& w_{2}(n+1)=w_{2}(n)+\mu_{c} e(n) \sin \left(\omega_{0} n\right)
\end{aligned}
$$

The performance of this sort of adaptive filters is generally superior to that of a fixed notch filter if it converges to the correct phase and amplitude. The convergence of the algorithm leads to cancellation of the corresponding narrow-band disturbance. A detailed analysis of this sort of systems is done in [1] and [3], where it is shown that using $\mathrm{Z}$ transform the following transfer function is obtained: 


$$
\begin{gathered}
\frac{Y(z)}{E(z)}=\mu_{c} \frac{z \cos \omega_{0}-1}{z^{2}-2 z \cos \omega_{0}+1}=H(z) \\
\frac{E(z)}{X(z)}=\frac{1}{1+G_{p}(z) H(z)}
\end{gathered}
$$

So, according to this formulation the closed-loop poles depend on the transfer function $G_{p}$. The XLMS algorithm compensates this effect by using a filtered reference sigual, but for low frequencies this compensation is not necessary [3]: if the sub-harmonics are into the pass-band of the vibrating system then the effect of the transfer function $G_{p}$ can be considered as a constant gain value $k_{p}$. In this case we approximate:

$$
G_{p}(z) H(z) \approx k_{p} \mu_{c} \frac{z \cos \omega_{0}-1}{z^{2}-2 z \cos \omega_{0}+1}
$$

Thus:

$$
\begin{gathered}
\frac{E(z)}{X(z)} \approx \frac{z^{2}-2 z \cos \omega_{0}+1}{z^{2}+z(\mu-2) \cos \omega_{0}+(1-\mu)}= \\
=\frac{\left(z-e^{j \omega_{0}}\right)\left(z-e^{-j \omega_{0}}\right)}{z^{2}+z(\mu-2) \cos \omega_{0}+(1-\mu)} \\
\text { where: } \mu=k_{p} \mu_{c}
\end{gathered}
$$

The previous transfer function corresponds to a second order notch filter which root-locus is a circle centered at the point $1 / \cos \omega_{o}$ of the abscises axis. This is shown in the following figure:

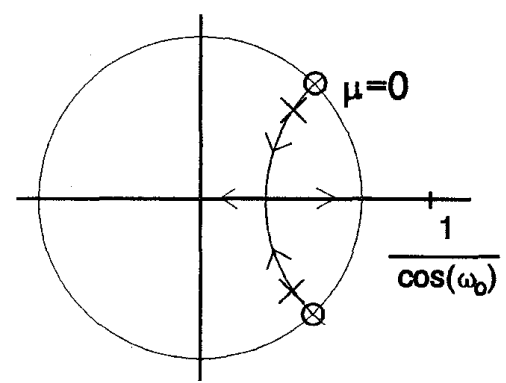

Fig. 4. Root locus.

The zeros are at the frequency $\omega_{0}$ for all $\mu$ values. If $0<\mu<2$ then the filter poles are inside the unit circle (stability condition). So, in order to have a stable narrowband notch filter the $\mu$ parameter must be sufficiently small. The poles can be fixed by adjusting $\mu$, thus providing the desired closed-loop frequency response: a small $\mu$ is related to a small notch bandwidth. However the filter adjustment is more critical for small $\mu$ values because in this case the canceller needs a precise reference signal, so it is required a best measurement of the frequency to cancel. In practice $\mu$ is selected according to the observed behaviour of the canceller.

\subsection{Cancelling Multiple Frequencies.}

In this case the canceller is a bank of notch filters, where each adaptive band-pass filter $H_{i}(z)$ centers on an unwanted sinusoidal signal (sub-harmonic) and exhibits a resonance at frequency $\omega_{i}$. The canceller has the form of a finite sum of second order adaptive notch filters, as is shown in figure 5 :

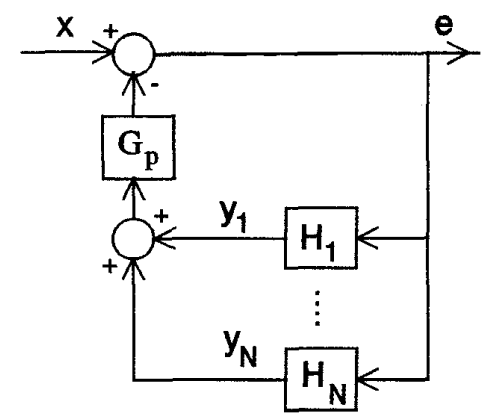

Fig. 5. Bank of notch filters.

So, the closed-loop transfer function is:

$$
\frac{E(z)}{X(z)}=\frac{1}{1+G_{p}(z) \sum_{i=1}^{N} H_{i}(z)}
$$

If the harmonics to be cancelled are inside the frequency response of $G_{p}$ we can consider $G_{p}(z) \propto k_{p}$. Thus:

$$
\frac{E(z)}{X(z)} \approx \frac{1}{1+k_{p} H_{1}(z)+\ldots+k_{p} H_{N}(z)}
$$

which zeros are at the frequencies $\omega_{\mathrm{i}}$. Moreover, due to the narrow bandwidth of each $H_{i}$ (small $\mu$ ) the closed-loop poles are approximately the same as in the case of each individual filter. The root-locus shown in figure 6 has been obtained from computer simulations of a canceller composed by four adaptive notch filters, tuned at the 
frequencies: $\omega_{1}=\pi / 10, \omega_{2}=2 \omega_{1}, \omega_{3}=3 \omega_{1}$, and $\omega_{4}=4 \omega_{1}$.

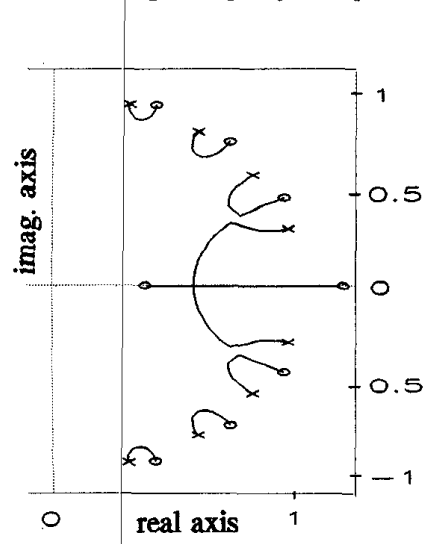

Fig. 6. Root-locus.

The frequency response of the previous filter is shown in the following figure $(\mu=0.05)$ :

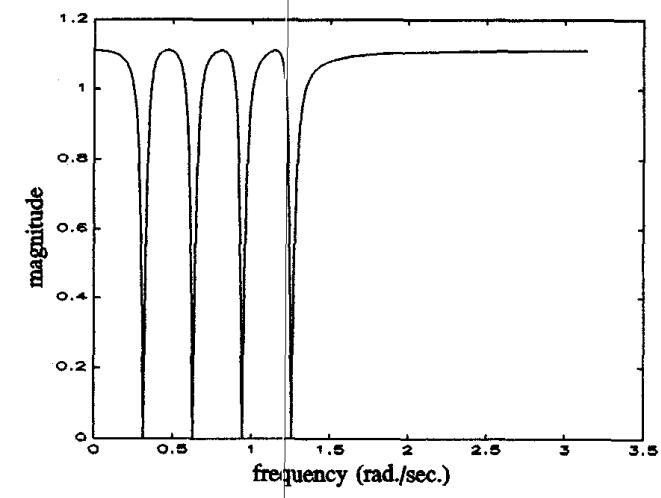

Fig. 7. Closed-loop frequency response.

Simnon simulations have been performed in order to evaluate the features of the canceller. For example, if the proposal is to cancel a disturbance as:

$$
\begin{gathered}
d=0.2 \cos \left(\omega_{1} n\right)+0.1 \sin \left(\omega_{1} n\right)+0.1 \cos \left(\omega_{2} n\right)+ \\
+0.3 \sin \left(\omega_{2} n\right)+0.1 \cos \left(\omega_{3} n\right)+0.06 \sin \left(\omega_{3} n\right)+ \\
\quad+0.07 \cos \left(\omega_{4} n\right)+0.02 \sin \left(\omega_{4} n\right)
\end{gathered}
$$

then, considering $\mu=0.05$ and $r=1$ the following graphical result of the error evolution have been obtained:

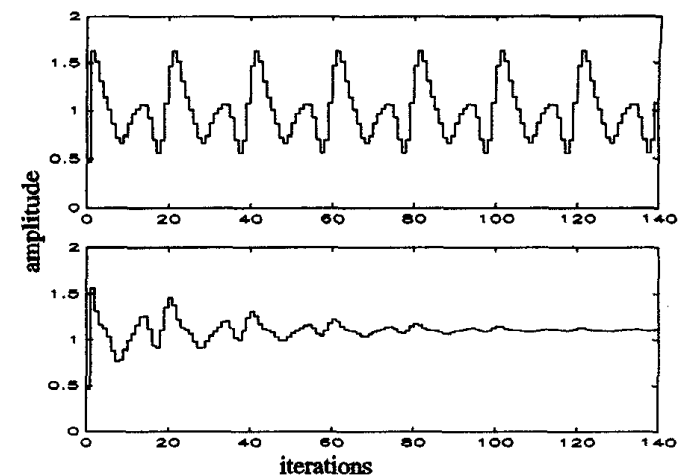

Fig. 8. Simnon simulation results. Signals: $\mathbf{x}$ (above) and $\mathrm{e}$ (below).

\section{Practical Results}

The developed canceller has been implemented using a PClabcard-812 included in a 486/DX2-66 PC, which sampling rate is close to 1000 Samples/second. The vibrating system chosen for benchmarking proposals has been a DC motor: its nominal rotation frequency is 7.2 $\mathrm{Hz}\left(f_{p}=7.2 \mathrm{~Hz}\right)$ and its bandwidth $4 \mathrm{~Hz}$.

The spectral analysis of the tachometer signal reveals four vibrating sub-harmonics (Figure 11) which frequencies are: $0.24 \mathrm{~Hz}, 0.48 \mathrm{~Hz}$ (it has the biggest amplitude), $0.72 \mathrm{~Hz}$ and $0.96 \mathrm{~Hz}$. So, the relations between the sub-harmonics and the nominal speed are: $f_{p} / 30,2 x\left(f_{p} / 30\right), 3 x\left(f_{p} / 30\right), 4 x\left(f_{p} / 30\right)$. When the proposed canceller is used the tachometer signal spectrum reveals an important reduction in the level of mechanical vibration (see Figure 12).

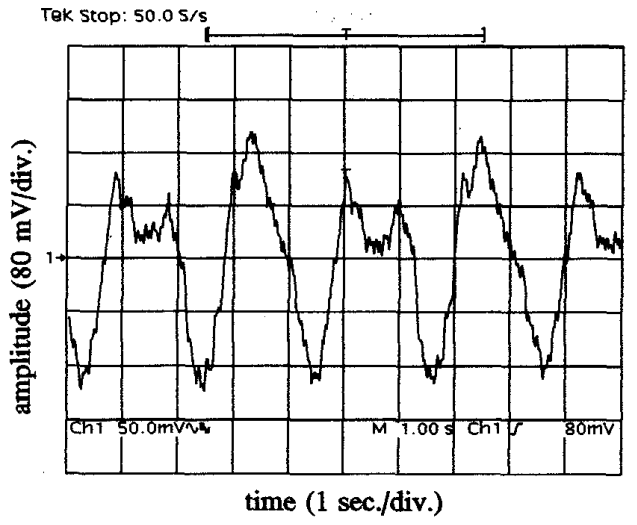

Fig. 9. Tachometer signal, without canceller. 


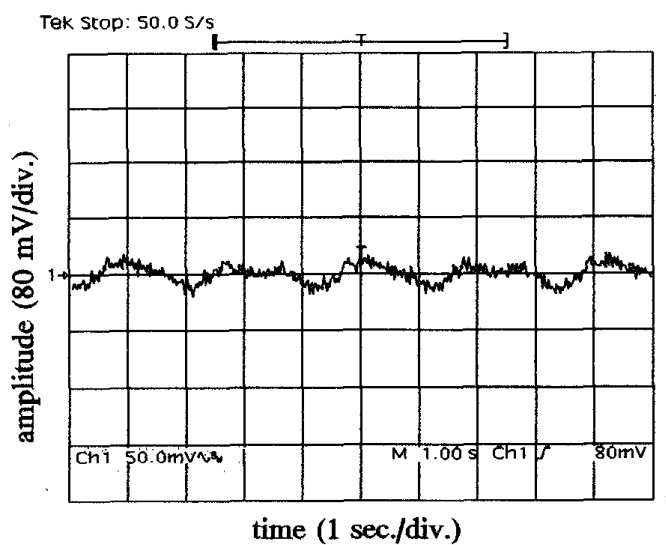

Fig. 10. Tachometer signal, with canceller.

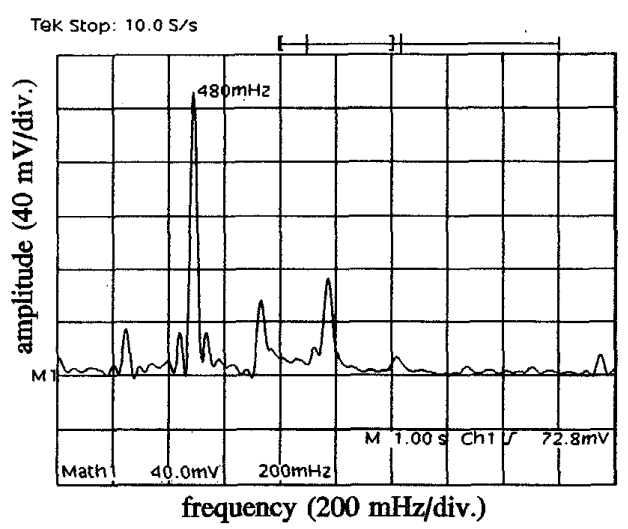

Fig. 11. Tachometer spectrum, without canceller.

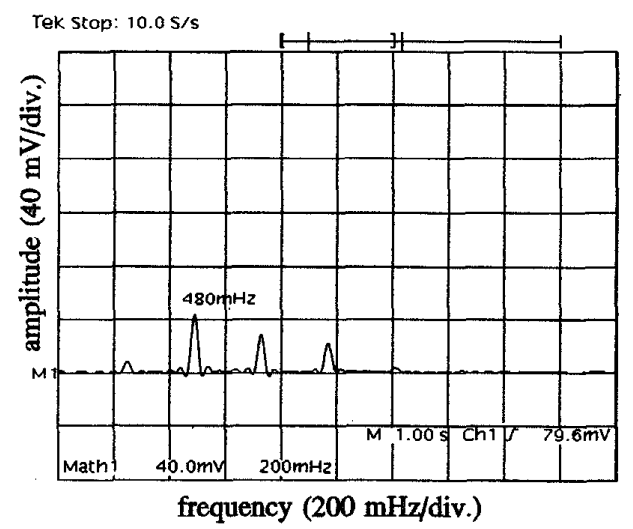

Fig. 12. Tachometer spectrum, with canceller.

\section{Conclusions}

The tachometer. signals shown in Fig.10 and Fig.12 (time-domain and spectrum, respectively) are obtained when the adaptive canceller is used. This experimental results reveal that a considerable vibration reduction is achievable. For instance, the main sub-harmonic $(0.48$ $\mathrm{Hz}$ ) is reduced $13.7 \mathrm{~dB}$.

\section{References}

[1] S.J. Elliot and P.A. Nelson. "Active Noise Control", IEEE Signal Processing Magazine, pp. 12-35 ,October 1993.

[2] R. Herzog et al. "Unbalance Compensation Using Generalized Notch Filters in the Multivariable Feedback of Magnetic Bearings", IEEE Transactions on Control Systems Technology, Vol. 4, pp. 580-586, no. 5, 1996.

[3] S.M. Kuo and M.J. Ji. "Development and Analysis of an Adaptive Noise Equalizer", IEEE Transactions on Speech and Audio Processing, Vol. 3, no. 3, pp. 217222, 1995.

[4] S.M. Kuo and D.R. Morgan, Active Noise Control Systems, John Wiley, 1996.

[5] L.A. Sievers and A.H. von Flotow. "Comparison and Extensions of Control Methods for Narrow-Band Disturbance Rejection", IEEE Transactions on Signal Processing, Vol. 40, no. 10, pp. 2377-2391, 1992. 